\title{
Changes in the body mass of adult residents of rural and urban areas in the initial months of the COVID-19 pandemic vs. their mental, physical and sexual health
}

\author{
Maciej Białorudzki ${ }^{1,2, A-F}$, Zbigniew Izdebski ${ }^{3,2, A-B, D-F} \oplus$ \\ ${ }^{1}$ Faculty Of Health Sciences, Medical University, Warsaw, Poland \\ 2 Department of Humanization of Health Care and Sexology, Collegium Medicum, University of Zielona Góra, Poland \\ ${ }^{3}$ Department of Biomedical Aspects of Development and Sexology, Faculty of Education, Warsaw University, Poland \\ A - Research concept and design, B - Collection and/or assembly of data, C - Data analysis and interpretation, \\ $D$ - Writing the article, $E$ - Critical revision of the article, F- Final approval of the article
}

Białorudzki M, Izdebski Z. Changes in the body mass of adult residents of rural and urban areas in the initial months of the COVID-19 pandemic vs. their mental, physical and sexual health. Ann Agric Environ Med. 2021; 28(4): 667-675. doi: 10.26444/aaem/143561

\begin{abstract}
Introduction and objective. A change in the body mass may be one of the health consequence of the COVID-19 pandemic, and may affect the health condition measured in many dimensions. The paper aims at assessment of the level and determinants of the body mass changes and stress level in the initial months of the COVID-19 pandemic, and the interrelation of these two factors.

Materials and method. Data were collected in June 2020, as a cross-sectional on-line survey. The representative sample of 2,535 Poles aged 18-65 was analysed. The main outcome is the Cohen's stress index PSS-4. Among thirteen independent variables, five were related to the change observed during the pandemic (including the body mass change and satisfaction with sexual life).

Results. Increase of the body mass within the period of the pandemic was declared by $33.9 \%$ of the respondents, including $36.1 \%$ in urban and $30.9 \%$ in rural areas $(p=0.026)$. The average increase of body mass was $5.11 \mathrm{~kg}$. The increase of body mass was related to the existing overweight and obesity, occurrence of chronic diseases, episodes of physical and mental crisis, and decrease of interest in sexual activity. The average index of stress in the initial months of the pandemic was $6.38 \pm 2.94$. Multivariate regression analysis showed eight independent predictors of stress in the whole group, seven in towns and five in rural areas. The significance of the relationship with the body mass increase was proved only among residents of rural areas.

Conclusions. The initial months of the COVID-19 pandemic were reflected, to a different extent, among residents of urban and rural areas. Body mass change and sexual health indicators remained significant predictors of stress level, even after analyses were corrected for other covariates.
\end{abstract}

\section{Key words}

stress, place of residence, social conditions, COVID-19, mental health, sexual health, body mass change

\section{INTRODUCTION}

The pandemic of the acute disease of respiratory system COVID-19 caused by the SARS-Cov-2 virus resulted in a worldwide health and economic crisis, and it also affected social life. Poland was among the countries where lockdown and gradual restrictions were soon introduced. The state of pandemic was announced on 11 March 2020, seven days after the first case had been detected in a patient from Zielona Góra, western Poland [1].

Specialized literature offers numerous articles concerning the influence of the pandemic on many aspects of health, including mental and sexual health. It is also emphasized how the period of the pandemic has changed the manner of performing work, family life, bonds in relationships, and other spheres of people's functioning. The processes might be analyzed at various stages of the pandemic. Articles

Address for correspondence: Maciej Białorudzki, Faculty Of Health Sciences, Medical University, Warsaw, Poland

E-mail: mbialorudzki@uz.zgora.pl

Received: 23.09.2021; accepted: 02.11.2021; first published: 17.11.2021 pertaining to the initial months of the pandemic still prevail in the literature, but more and more papers concern further waves of the pandemic. Within the scope of health, apart from the obvious consequences of coronavirus infection, authors emphasize disadvantageous changes of lifestyle, including increased use of psychoactive substances and more seldom pro-health behaviours. Limited mobility, decreased physical activity in the leisure time and changes in eating habits might result in weight gain. Increased stress was an additional risk factor. It may be assumed that the relation of stress and changes of body mass is of a mutual nature.

Mental problems are less characteristic of the COVID-19 pandemic period $[2,3]$. The restrictions, less stable labour market, uncertainty of the future, risk of the disease and inconsistent information published by the media, were a source of permanent stress. Psychological results of the pandemic also include fear, anger, depression, frustration and even symptoms of post-traumatic stress $[4,5]$. Particularly in the initial months of the pandemic, society was overloaded with information which was not always authentic and verified. Anxiety and stress might be heightened by this 
general disinformation, and also by a healthcare system not adapted to combating the pandemic, and terrifying statistical data concerning the number of COVID-19 cases and fatalities [6].

The COVID-19 pandemic confirmed that stressful events affect nutrition patterns [7]. Depending on whether the stress is acute or chronic, it may cause hypofagia or hyperfagia and binging, which eventually leads to a significant change of body mass $[8,9,10]$. Stress, depression symptoms, financial problems and pronounced loneliness during the COVID-19 pandemic might cause episodes of increased eating and binging, used to cope with and regulate emotions [11].

The influence of the pandemic on motivation to reduce body mass by overweight people is a separate question. Almandoz et al. indicated in their research that $69.6 \%$ of patients considered objectives related to weight loss as more difficult to achieve during the COVID-19 period [12]. There is also evidence that eating habits were characterized by higher energy consumption and lower nutritive quality during the pandemic than before COVID-19. In Spain, people consumed, on average, $539 \mathrm{kcal}$ more than recommended [13]. It was also proved that $43.5 \%$ of respondents ate more during quarantine than before, and $51.8 \%$ admitted they snacked more often between meals. Moreover, the higher consumption of calories and snacking were more often reported by people with a higher BMI [14]. The pandemic also affected access to food and consumption behaviours - $49.6 \%$ declared they were storing more food than usual, and $61.2 \%$ ate more than usual due to stress [12]. It was also shown that during the COVID-19 pandemic adults reported a higher, unrestrained appetite, overeating and consumption of unhealthy food to cope with stress $[15,16,17]$.

The pandemic has also significantly affected the quality of life, including the sphere of sexual life. The social distancing caused by COVID-19 might negatively affect sexual activity of the respondents, which concerned both the bonds in relationships and beyond, including problems of sexual minorities $[18,19]$. Research on sexual life include, in a broad sense, examination of the individual's physical, emotional, mental, and social condition with reference to sexuality beyond the absence of a disease, dysfunctions, or disabilities [20]. It seems justified to include the assessment of satisfaction with sexual life in research on health condition of the society performed in the pandemic era. Guanjian Li et al. showed the direct influence of COVID-19 on sexual health, which was manifested by a decrease in sexual drive and lower frequency of sexual intercourses [21]. A Polish prospective observational study showed an increase in the sexual dysfunctions in Polish women during the COVID-19 pandemic, from $15.3 \%$ to $34.3 \%$ [22]. Again, it seems justified to relate the information about problems with mental health, changes in health behaviour and changes of body mass with the assessment of sexual life. In both men and in women obesity may be a factor that impedes sexual life, and living in stress strengthens the difficulties. It was found that the frequency of intercourse was lower in overweight people, desire was reduced, and no sexual satisfaction was experienced [23].

While analyzing the foregoing three aspects of functioning during the COVID-19 pandemic (mental health, changes of body mass and assessment of sexual life), it is worth mentioning the wider social context and the effect of place of residence. A hypothesis may be assumed that residents of towns of various sizes and residents of rural areas in Poland were, to a different extent, exposed to the economic, social and health results of the pandemic. It may be expected that the results of the pandemic were less perceptible in rural areas.

To the best of the knowledge of the authors of the current study, no reports have been published that analyze jointly the aspect of unfavourable changes of body mass with the assessment of mental health and sexual life in the period of COVID-19 in Poland, and which also consider the differences between residents of urban and rural areas. To this end, a large population survey of June 2020 is a unique source of data. It is also one of the few Polish surveys that broadly describes the functioning of society in the initial months of the pandemic, and provides comparison of the various social groups.

\section{OBJECTIVE}

The analyses were aimed mainly at presenting the relationship between changes of body mass, and the physical, mental, and sexual health of Poles within the period from March - June 2020, i.e., in the initial months of the COVID-19 pandemic. The Cohen stress index was assumed as the major outcome measure, with the change of body mass as the main predictor. Social determinants of both factors were presented. Apart from the change of body mass, the conditions of stress included several social and demographic variables, BMI, prevalence of chronic diseases, and other indicators of mental well-being. Several indicators and relations were presented as divided into urban and rural environments. A hypothesis was assumed that the place of residence was a factor that diversified the reaction to the initial months of the COVID-19 pandemic. The following research questions were asked:

1) How often did the respondents from urban and rural areas report a change of body mass in the initial months of the pandemic?

2) What factors affected the change of body mass?

3) What was the intensity of stress among adult residents of urban and rural areas in the initial months of the COVID-19 pandemic?

4) What factors affected changes in the stress level?

5) Were changes of body mass and stress level related to the assessment of sexual life?

6) Do the change of body mass and individual perception of sexual life remain significant predictors of the stress level after other analyzed factors have been considered?

\section{MATERIALS AND METHOD}

Respondents. The data was collected within the framework of the project "Health, bonds in relationships and sexual life of Poles in the era of COVID-19" conducted by Z. Izdebski et al. The questionnaire survey was conducted using the Internet method (CAWI) at end of May and beginning of June 2020. The respondents were from the all-Poland panel sample of 100,000 persons who participated in various Internet surveys conducted by an external contractor (IQS Polska). A representative for the country structure of the sample was assumed according to gender, age, and place of residence (province, size of town). The survey was conducted 
in a representative all-Poland sample of 3,000 persons aged 18-86 (age median: 45 years). A subgroup of respondents was selected for the current analysis $(\mathrm{N}=2,535)$. The main criterion for inclusion was age $18-65$ years and completeness of the information about stress, changes of body mass and BMI level. The survey was approved by the Commission of Research Studies Ethics of the University of Warsaw (Opinion No. 6/2020).

Questionnaire. The full research tool was a questionnaire that included about 500 variables in 16 thematic sections. The time limit to complete the questionnaire was a maximum 25 minutes, and some questions and groups of questions were included optionally, depending on previous answers. A significant number of questions concerned the past 2-3 months when strict limitations were introduced in Poland, with reference to social isolation.

Change of body mass. Following the main aim of the study, the subjective change of body mass was assumed as the central variable. The question was asked: 'Within the past 2-3 months, how did your weight/body mass change?', and the following answers might be selected: 'it increased'; it did not change'; 'it decreased'. The respondents were also asked about their body mass and height, which allowed calculation of the current BMI index according to the self-report data. The respondents also estimated the increase of their body mass in kilograms, compared to the period preceding the pandemic. Depending on the stage of the analyses, the change of body mass was considered as a dependent or independent variable.

Mental health. The average Cohen stress index from the PSS- 4 scale was assumed as the main dependent variable. The scale is comprised of four questions from the point of view of experience of the past month. The questions come from the original larger tool PSS-14. An example of such questions is: 'In the past month, how often have you felt difficulties were piling up so high that you could not overcome them?' Five categories of answers were provided, i.e., from 'never' to 'very often'. The answers were on a scale from $0-4$ for negative and from 4-0 for positive statements. The summary index ranged from 0-16 points, where a high score means a significant intensity of stress. In the surveyed sample, the stress index had a univariate structure; Cronbach's coefficient -0.581 . The relatively poor reliability of PSS-4 is reported in other surveys and explained by the presence of counter-oriented questions [24].

The sphere of mental health may also include subjectively perceived mental crisis. The question was asked: 'Within the past 2 - months of the coronavirus pandemic, did you have a period of collapse, mental crisis?' The possible answers were: 'Yes, I still do'; 'yes, but it's gone'; 'no, I did not experience it'.

Sexual health. To assess changes in sexual health, the question about the decrease of interest in sex was chosen, i.e. 'Within the past 2-3 months, did you notice your longterm decrease of interest in sex?'. The possible answers were: 'Yes', or 'No'. There was also a question about the general assessment of sexual life: 'Within the scope of the past 2-3 months, how do you assess various spheres of your life: your sexual life?' There were five answers to choose from, ranging from 'Very bad' to 'Very good'.
Other covariates. Among the analyzed covariates there were social and demographic features, such as: gender, age, place of residence, level of education, whether in a relationship, occupational activity, and other covariates related to health, e.g., any chronic diseases or crisis of physical health during the pandemic. The physical crisis was defined as a period of fatigue or exhaustion. Following preliminary analyses, some covariates were re-coded into a smaller number of categories, compared to the source version of the questionnaire. While examining the status of a relationship, formal (marriage) and informal relationships were analyzed together. Occupational activity was re-coded from 10 detailed categories of employment status, and the education level from 12 more detailed categories. Seven categories of the size of places of residence were re-coded to a simple division between urban and rural areas.

Statistical analyses. The relation between categorized covariates was examined with the chi-square test. The average values (SD) of the Cohen stress index in various groups of respondents were indicated by testing the differences with Mann-Whiney non-parametric tests (two groups) or KruskalWallis test (comparison of more than two groups). The measure of the difference in the strength of the correlation of stress with individual factors in urban and rural areas was the eta coefficient as estimated from ANOVA.

Multivariate models of linear regression were estimated with the stress index as a dependent variable, separately in total sample, and for residents of urban and rural areas. The findings were presented as standardized beta regression coefficients. The quality of the model fit was evaluated using the R-squared coefficient of determination. The level of significance was assumed as $\mathrm{p}<0.05$ for all tests. Analyses were performed with the IBM SPSS Statistics v. 27 software.

\section{RESULTS}

1. Characteristics of the sample. The basic social and demographic characteristics of the 2,535 respondents aged 18-65 who took part in the Internet surveys are presented in Table 1. The group was balanced for gender (51.6\% women); average age -41.73 years $(S D=13.82)$, and the median was close to the average (41 years). The largest group included people aged $30-49$. In line with the inclusion criterion, the age ranged from 18-65 years. The structure of the sample according to the place of residence was close to the all-Poland data with $42.7 \%$ representation of residents of rural areas. Among the respondents, 44.9\% people had lower than secondary education, and $21.9 \%$ of the respondents reported university education. Three quarters of the surveyed group were in regular relationships, and most participants were employed (58.9\%). The differences arising from the place of residence were as follows: in urban areas, there was a significantly higher proportion of male respondents $(p<0.001)$, occupationally active $(p=0.035)$, and those with university education $(\mathrm{p}<0.001)$. The structure according to the three age groups and the status of relationship did not differ between the two categories of places of residence ( $\mathrm{p}=0.067$ and $\mathrm{p}=0.366$ respectively).

Similarly, Table 2 presents the distribution of answers to the questions concerning physical and mental health condition, 
Table 1. Selected social and demographic features of respondents according to place of residence

\begin{tabular}{lcccccc}
\hline & \multicolumn{2}{c}{$\begin{array}{c}\text { Total } \\
\text { N=2535 }\end{array}$} & \multicolumn{2}{c}{$\begin{array}{c}\text { Urban } \\
\mathrm{N}=1453\end{array}$} & \multicolumn{2}{c}{$\begin{array}{c}\text { Rural } \\
\text { N=1082 }\end{array}$} \\
\cline { 2 - 8 } & $\mathrm{N}$ & $\%$ & $\mathrm{~N}$ & $\%$ & $\mathrm{~N}$ & $\%$ \\
\hline Gender & 1228 & 48.4 & 753 & 51.8 & 475 & 43.9 \\
\hline Male & 1307 & 51.6 & 700 & 48.2 & 607 & 56.1 \\
\hline Female & & & & & & \\
\hline Age (years) & 540 & 21.3 & 324 & 22.3 & 216 & 20.0 \\
\hline $18-29$. & 1147 & 45.2 & 629 & 43.3 & 518 & 47.9 \\
\hline $30-49$ & 848 & 33.5 & 500 & 34.4 & 348 & 32.1 \\
\hline$\geq 50$ & & & & & & \\
\hline Level of education & 1122 & 44.3 & 590 & 40.6 & 532 & 49.2 \\
\hline Lower than secondary & 857 & 33.8 & 515 & 35.4 & 342 & 31.6 \\
\hline Secondary & 556 & 21.9 & 348 & 24.0 & 208 & 19.2 \\
\hline Higher than secondary & & & & & & \\
\hline Union status & 613 & 24.2 & 361 & 24.9 & 252 & 23.3 \\
\hline Single & 1922 & 75.8 & 1092 & 75.2 & 830 & 76.7 \\
\hline Married or cohabiting & 1447 & 58.9 & 850 & 60.7 & 597 & 56.5 \\
\hline Employment status & 1010 & 41.1 & 550 & 39.3 & 640 & 43.5 \\
\hline Working & & & & & & \\
\hline Not working & & & & & & \\
\hline & & & & & & \\
\hline
\end{tabular}

Table 2. Selected indicators $f$ sexual health and life of the respondents according to their place of residence

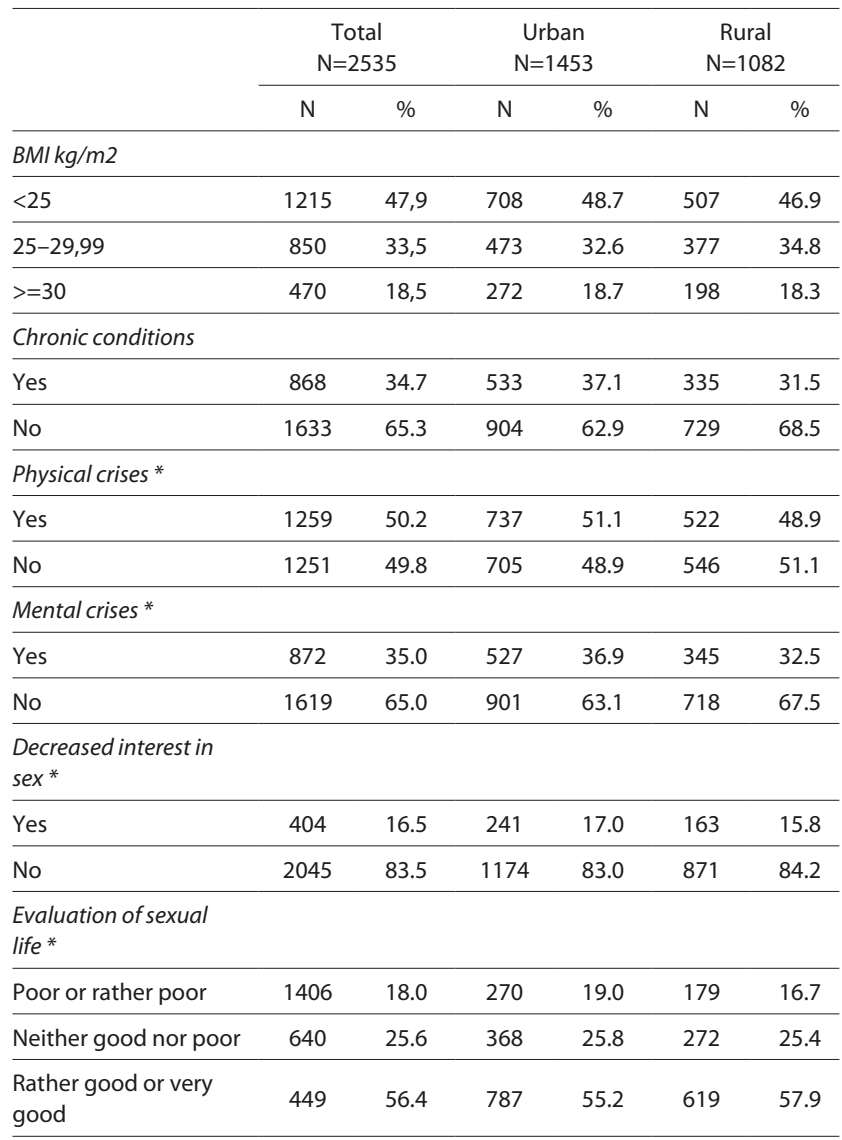

* The question concerned the past 2-3 months as well as the assessment of sexual life. Four questions referred to the changes in the period of the pandemic, i.e., the past 2-3 months. No overweight was found in nearly half of the respondents, including 3.5\% with underweight and $44.4 \%$ with normal body mass. Almost one-in-five (18.5\%) of the respondents was obese with BMI higher than $30 \mathrm{~kg} / \mathrm{m} 2$. More than one-third of the respondents reported at least one chronic disease diagnosed by a physician. Taking the experience of the period of the pandemic into account, at least one episode of a physical or mental crisis was reported by most respondents (57.5\%). Episodes of physical exhaustion were reported more often than mental rexhaustion. Most of the surveyed Poles aged 18-65 assessed their sexual life within the past 2-3 months as good; however, $16-18 \%$ had problems, and evaluated their sexual life as much worse, or reported a decrease of interest in sex within that period.

While comparing the residents of urban and rural areas, no differences were found in the current distribution of body mass measured with BMI ( $\mathrm{p}=0.477)$. Residents of urban and rural areas did not differ in the occurrence of episodes of physical crises $(p=0.269)$, whereas mental crises were reported more often in urban than in rural areas $(\mathrm{p}=0.021)$, along with the occurrence of chronic diseases $(p=0.007)$.

2. Respectively, whereas the differences of the proportion of those who reported decrease of body mass was only slight (15.2\% vs. $16.3 \%)$, the average increase and decrease of body mass estimated in kilograms was similar (5.11 \pm 3.71 and $5.37 \pm 3.92 \mathrm{~kg}$, respectively).

More advanced analyses were performed for each gender separately. Increase of body mass was declared more often by women than by men $(38.0 \%$ vs. $29.5 \%$; $\mathrm{p}<0.001)$. Differences of body mass changes related to the place of residence were more frequent among men $(\mathrm{p}=0.034)$ than among women $(p=0.075)$. The level of body mass increase measured in kilograms was similar in both urban and in rural areas (5.22 \pm 3.77 vs. $5.00 \pm 3.67 ; \mathrm{p}=0.919)$, but only in the group of men. Women who lived in urban areas declared a lower increase of body mass than women who lived in rural areas (4.73 \pm 3.54 vs. $5.55 \pm 4.02 ; \mathrm{p}=0.016$ ).

Body mass changes were analyzed in detail by comparison of the groups of respondents distinguished by social and demographic features, health condition and assessment of sexual life. Apart from the factors of gender and place of residence discussed above, age and education are worth mentioning as differentiating factors. The proportion of persons whose body mass increased was lower in the group of respondents with lower than secondary education (30.7\%), than it was among the respondents with secondary (36.3\%) and university $(36.5 \%)$ education $(\mathrm{p}=0.038)$. The profile of body mass changes was different among younger respondents aged 18-29 than from among those aged 30-49 and 50+ $(\mathrm{p}<0.001)$. A higher proportion of those who declared both increase (39.4\%) and decrease of body mass (21.1\%) was found in the group of younger respondents.

Table 3 presents body mass changes according to six features related to physical and mental health, and to assessment of sexual life; Figure 1 presents the selected data separately for urban and rural areas. Only general assessment of sexual life was not related to body mass changes $(p=0.111)$. In the initial period of the pandemic, increase of body mass was more often found in persons with overweight and obesity, 
Table 3. Body mass change within the past $2-3$ months according to selected indicators of sexual health and life

\begin{tabular}{|c|c|c|c|c|}
\hline & \multicolumn{3}{|c|}{ Change in body weight } & \multirow{2}{*}{$\mathrm{p}$} \\
\hline & Increase & No change & Decrease & \\
\hline Total & 33.9 & 50.4 & 15.7 & \\
\hline \multicolumn{5}{|l|}{$B M / \mathrm{kg} / \mathrm{m} 2$} \\
\hline$<25$ & 28.0 & 54.6 & 17.3 & \\
\hline $25-29,99$ & 36.7 & 50.0 & 13.3 & $<0.001$ \\
\hline$>=30$ & 43.8 & 40.4 & 15.7 & \\
\hline \multicolumn{5}{|l|}{ Chronic conditions } \\
\hline Yes & 37.9 & 45.5 & 16.6 & 0.002 \\
\hline No & 32.0 & 52.8 & 15.2 & \\
\hline \multicolumn{5}{|l|}{ Physical crises * } \\
\hline Yes & 39.1 & 43.2 & 17.8 & $<0.001$ \\
\hline No & 28.8 & 57.5 & 13.7 & \\
\hline \multicolumn{5}{|l|}{ Mental crises * } \\
\hline Yes & 39.2 & 40.5 & 20.3 & $<0.001$ \\
\hline No & 31.4 & 55.3 & 13.3 & \\
\hline \multicolumn{5}{|l|}{ Decreased interest in sex * } \\
\hline Yes & 40.8 & 44.3 & 14.9 & 0.006 \\
\hline No & 32.6 & 51.5 & 15.9 & \\
\hline Poor or rather poor & 38.9 & 45.0 & 16.1 & \\
\hline Neither good nor poor & 33.1 & 50.9 & 16.0 & 0.111 \\
\hline Rather good or very good & 32.6 & 51.9 & 15.5 & \\
\hline
\end{tabular}

* The question concerned the past 2-3 months; $p$ in the independence chi-square test

and in those with at least one chronic disease. The body mass increase was also related to episodes of physical and mental crisis, and to decreased interest in sexual activity.

The statistically significant correlation between BMI level and occurrence of physical crisis was maintained in urban and rural areas (Fig. 1), whereas the conclusions for other factors are ambiguous. A negative assessment of sexual life was connected to increase of body mass in the period of the pandemic more often in rural areas than in towns, while the occurrence of a chronic disease and mental crises were stronger predictors of body mass changes in urban than in rural areas.

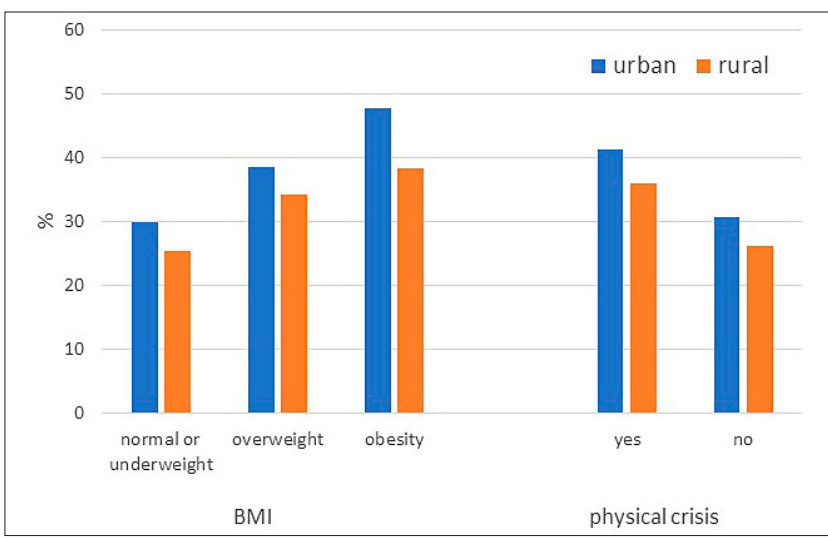

Figure 1. Respondents who gained weight during the pandemic period (\%) according to the place of residence, BMI level and physical crisis episodes
3) Stress level in Cohen scale. In the surveyed group of adult Poles, the average stress index was $6.38 \pm 2.94$ The difference between residents of urban and rural areas was not statistically significant $(\mathrm{p}=0.302)$. Table 4 shows the correlation of the selected social and demographic features with the level of stress in the general group of respondents and, separately, among residents of towns and rural areas. Men felt a significantly lower level of stress than women, and this difference was stronger in rural than in urban areas. The average level of stress decreased in consecutive age groups. In the whole group and among residents of towns, a systematic improvement with the age was observed whereas in rural areas, the improvement concerned only people older than 50 . The correlation is thus stronger among residents of urban, compared to residents of rural areas.

Single people felt higher levels of stress than those who lived in relationships. The difference was statistically significant, both for residents of urban and rural areas; however, the correlation was stronger in rural areas. The Cohen index was about 0.88 points higher for single people than people living in relationships, and in urban areas the difference was 0.59 . It was also found that occupationally active people feel a lower stress level than those who were inactive. In this case, the correlation was stronger in urban than in rural areas.

Education was the last factor analyzed. A statistically significant correlation was found in the whole group and among residents of rural areas; however, the linearity of the correlation was not found. A lower stress level was observed among people with university education, whereas the respondents with secondary education or lower showed a similar score in the Cohen scale. The difference between the extreme groups was 1.00 in residents of rural areas, compared to 0.39 for urban residents $(p=0.135)$. The strength of the correlation measured with eta coefficient was more than twice higher in rural areas ( 0.130 vs. 0.053). The analyses presented in Table 4 show that in rural areas the correlation between the stress level and the relation status or education level was stronger, but the correlation with occupational activity was not significant. In urban areas, only the correlation between stress level and education level was not significant.

Table 5 shows the association between physical, mental, and sexual health and the stress level, in the whole group and divided into residents of urban and rural areas.

Within the factors related to physical health, the correlation with the BMI level appeared significant, although it was not of linear nature. A significant relationship was found only in towns. Overweight people reported the lowest stress levels. In rural areas, the stress level even decreased as the BMI increased. Absence of body mass changes was related to a lower average stress level, whereas the Cohen index grew with increase or decrease of body mass. The association of stress with body mass was more vivid in rural areas. People with chronic diseases reported higher stress than healthy ones, but a significant correlation was proved only in urban areas. A significant correlation of stress with episodes of physical fatigue or exhaustion was also confirmed only in urban areas.

A relationship with stress level was also proved for the group of factors related to physical and sexual health. The Cohen index was higher among the respondents who 
Table 4. Average Cohen stress indices according to social and demographic feature.

\begin{tabular}{lccc}
\hline Variable & Total & Urban & Rural \\
\hline Total & $6.38 \pm 2.94$ & $6.44 \pm 2.95$ & $6.29+2.94$ \\
\hline Gender & $6.56 \pm 3.02$ & $6.62 \pm 3.07$ & $6.49 \pm 2.95$ \\
\hline Female & $6.19 \pm 2.86$ & $6.28 \pm 2.83$ & $6.03 \pm 2.90$ \\
\hline Male & $\mathbf{0 . 0 0 1 / 0 . 0 6 4}$ & $\mathbf{0 . 0 3 9 / 0 . 0 7 8}$ & $\mathbf{0 . 0 3 1 / 0 . 0 5 8}$ \\
\hline p/eta & & & \\
\hline Age (years) & $6.89 \pm 3.15$ & $7.12 \pm 3.05$ & $6.55+3.27$ \\
\hline 18-29 & $6.50 \pm 2.78$ & $6.53 \pm 2.83$ & $6.46+2.71$ \\
\hline 30-49 & $5.90 \pm 2.96$ & $5.91 \pm 2.94$ & $5.89+3.00$ \\
\hline$\geq 50$ & $\mathbf{0 . 0 0 6 / 0 . 0 8 2}$ & $<\mathbf{0 . 0 0 1 / 0 . 1 5 3}$ & $\mathbf{0 . 0 0 7 / 0 . 0 9 6}$ \\
\hline p/eta & & & \\
\hline Union status & $6.21 \pm 2.91$ & $6.30 \pm 2.91$ & $6.09 \pm 2.91$ \\
\hline Married or cohabiting & $6.92 \pm 2.98$ & $6.89 \pm 3.02$ & $6.97 \pm 2.91$ \\
\hline Single & $<\mathbf{0 . 0 0 1 / 0 . 1 0 4}$ & $\mathbf{0 . 0 0 2 / 0 . 0 8 7}$ & $<\mathbf{0 . 0 0 1 / 0 . 1 2 6}$ \\
\hline p/eta & & & \\
\hline Employment status & $6.54 \pm 2.83$ & $6.56 \pm 2.84$ & $6.53 \pm 2.82$ \\
\hline Working & $6.17 \pm 2.88$ & $6.21 \pm 2.88$ & $6.10 \pm 2.88$ \\
\hline Not working & $\mathbf{6 . 0 0 1 / 0 . 0 7 6}$ & $\mathbf{0 . 0 0 2 / 0 . 0 8 3}$ & $0.061 / 0.068$ \\
\hline p/eta & $\mathbf{0 . 0 0 6 / 0 . 0 8 2}$ & $0.201 / 0.053$ & $<\mathbf{0 . 0 0 1 / 0 . 1 3 0}$ \\
\hline Level of education & & & $6.40 \pm 2.94$ \\
\hline Sower than secondary & & & \\
\hline Higher than secondary & & & \\
\hline peta & & & \\
\hline
\end{tabular}

* significance level ' $p$ ' corresponds to non-parametric Mann-Whiney or Kruskal-Wallis tests; eta is a measure of effect size in ANOVA

reported episodes of mental crises, compared to those who did not experience such episodes. Decreased interest in sex correlated with a higher stress level in the whole group, both in urban and rural areas. A similar conclusion pertained to the association of stress and general assessment of sexual life - stronger in rural areas.

Significance level 'p' corresponds to non-parametric MannWhiney or Kruskal-Wallis tests; eta is a measure of effect size in ANOVA; ${ }^{*}$ The question concerned the past 2-3 months

Table 6 presents a multivariate model of linear regression. The endogenous (dependent) variable is the Cohen stress level, the social and demographic features as well as the selected indicators of physical, mental, and sexual health, were considered as the exogenous (independent) variables.

Six significant factors may be distinguished as those which explain the stress level in towns, i.e., education level, occurrence of a chronic disease, mental and physical crisis, decrease of interest in and assessment of sexual life. It was also shown that in this linear model, the stress index decreased systematically with age, being the seventh significant predictor. In the model estimated for residents of rural areas, five significant factors were identified: education level, occurrence of a mental or physical crisis, assessment of sexual life and increase of body mass. Only the latter was not a significant predictor of changes of the stress level in towns.

In the general model, estimated for the whole sample and adjusted for the place of residence, there were eight significant factors. The new factor which was absent in the foregoing models was a weak correlation of stress levels with the decrease of body mass during the pandemic $(\mathrm{p}=0.042)$.
Table 5. Average Cohen stress indices according to the selected factors of physical, mental and sexual health.

\begin{tabular}{|c|c|c|c|}
\hline & Total & Urban & Rural \\
\hline Total & $6.38 \pm 2.94$ & $6.44 \pm 2.95$ & $6.29+2.94$ \\
\hline \multicolumn{4}{|l|}{$B M I \mathrm{~kg} / \mathrm{m} 2$} \\
\hline$<25$ & $6.59 \pm 2.98$ & $6.63 \pm 2.95$ & $6.47+3.02$ \\
\hline $25-29,99$ & $6.13 \pm 2.85$ & $6.11 \pm 2.92$ & $6.17+2.76$ \\
\hline$>=30$ & $6.33 \pm 3.00$ & $6.53 \pm 2.97$ & $6.07+3.03$ \\
\hline p/eta & $0.006 / 0.065$ & $0.018 / 0.079$ & $0.185 / 0.059$ \\
\hline \multicolumn{4}{|l|}{ Change in body weight * } \\
\hline Increase & $6.74 \pm 2.86$ & $6.67 \pm 2.91$ & $6.84 \pm 2.78$ \\
\hline No change & $5.99 \pm 2.91$ & $6.10 \pm 2.88$ & $5.86 \pm 2.96$ \\
\hline Decrease & $6.80 \pm 3.08$ & $6.95 \pm 3.17$ & $6.61 \pm 2.95$ \\
\hline p/eta & $<0.001 / 0.130$ & $<0.001 / 0.115$ & $<0.001 / 0.155$ \\
\hline \multicolumn{4}{|l|}{ Gender } \\
\hline Yes & $6.68 \pm 2.95$ & $6.80 \pm 2.97$ & $6.48 \pm 2.90$ \\
\hline No & $6.20 \pm 2.94$ & $6.23 \pm 2.93$ & $6.16 \pm 2.96$ \\
\hline p/eta & $<0.001 / 0.077$ & $0.001 / 0.093$ & $0.111 / 0.050$ \\
\hline \multicolumn{4}{|l|}{ Physical crises * } \\
\hline Yes & $7.15 \pm 2.71$ & $7.24 \pm 2.73$ & $7.03 \pm 2.68$ \\
\hline No & $5.58 \pm 2.98$ & $5.61 \pm 2.96$ & $5.54 \pm 3.01$ \\
\hline p/eta & $<0.001 / 0.267$ & $0.001 / 0.276$ & $<0.001 / 0.253$ \\
\hline \multicolumn{4}{|l|}{ Mental crises * } \\
\hline Yes & $7.96 \pm 2.51$ & $8.05 \pm 2.53$ & $7.83 \pm 2.47$ \\
\hline No & $5.51 \pm 2.81$ & $5.50 \pm 2.77$ & $5.52 \pm 2.86$ \\
\hline p/eta & $<0.001 / 0.397$ & $<0.001 / 0.417$ & $<0.001 / 0.367$ \\
\hline \multicolumn{4}{|l|}{ Decreased interest in sex * } \\
\hline Yes & $7.48 \pm 2.75$ & $7.60 \pm 2.77$ & $7.30 \pm 2.73$ \\
\hline No & $6.15 \pm 2.95$ & $6.19 \pm 2.95$ & $6.08 \pm 2.95$ \\
\hline p/eta & $<0.001 / 0.167$ & $<0.001 / 0.178$ & $<0.001 / 0.150$ \\
\hline \multicolumn{4}{|l|}{ Evaluation of sex life * } \\
\hline Poor or rather poor & $7.57 \pm 2.83$ & $7.65 \pm 2.77$ & $7.52 \pm 2.87$ \\
\hline Neither good nor poor & $6.86 \pm 2.66$ & $6.81 \pm 2.54$ & $6.90 \pm 2.75$ \\
\hline Rather good or very good & $5.78 \pm 2.95$ & $5.66 \pm 2.96$ & $5.87 \pm 2.93$ \\
\hline p/eta & $<0.001 / 0.245$ & $<0.001 / 0.115$ & $<0.001 / 0.155$ \\
\hline
\end{tabular}

In further analyses, it is worth looking at factors for which a borderline statistical significance was obtained, such as gender and employment status in urban areas.

Taking parameters of the linear value into account, factors that decreased the stress level were age, university education and positive assessment of sexual life. Other significant factors which increased the stress level in at least one of the three models were body mass changes, occurrence of a chronic disease and mental or physical crises during the pandemic.

Taking the absolute parameters of the linear regression into consideration, the mental crises noted during the pandemic were the strongest predictor of the increase of stress, and satisfaction with sexual life was the weakest predictor of decrease of stress. The negative influence of mental crises was stronger in urban areas (Tab. 6).

The R-sq indicators are the measure of the quality of models fit to the actual data. They depict the proportion of variability of the stress index explained by the analyzed factors, and reached a higher value in urban than in rural areas $(24.1 \%$ vs. $21.7 \%)$. 
Table 6. Linear regression models that explain the stress level

\begin{tabular}{|c|c|c|c|c|c|c|}
\hline \multirow{2}{*}{ Independent variable } & \multicolumn{2}{|c|}{ Total } & \multicolumn{2}{|c|}{ Urban } & \multicolumn{2}{|c|}{ Rural } \\
\hline & Beta & $p$ & Beta & $p$ & Beta & $p$ \\
\hline Place of living (urban) & 0.010 & 0.578 & - & - & - & - \\
\hline Gender (male) & 0.024 & 0.231 & 0.043 & 0.094 & -0.006 & 0.852 \\
\hline Age (cont.) & -0.062 & 0.003 & -0.086 & 0.002 & -0.021 & 0.521 \\
\hline Education (oder) & -0.076 & 0.000 & -0.066 & 0.007 & -0.094 & 0.002 \\
\hline Employment (0-1) & -0.037 & 0.057 & -0.046 & 0.068 & -0.023 & 0.438 \\
\hline Living single (0-1) & -0.012 & 0.549 & -0.048 & 0.077 & 0.039 & 0.216 \\
\hline BMI (cont.) & -0.033 & 0.106 & -0.030 & 0.261 & -0.039 & 0.203 \\
\hline $\begin{array}{l}\text { Increase of weight } \\
(0-1)\end{array}$ & 0.059 & 0.004 & 0.020 & 0.456 & 0.113 & 0.000 \\
\hline $\begin{array}{l}\text { Decrease of weight } \\
(0-1)\end{array}$ & 0.040 & 0.042 & 0.036 & 0.166 & 0.049 & 0.111 \\
\hline $\begin{array}{l}\text { Chronic conditions } \\
(0-1)\end{array}$ & 0.037 & 0.062 & 0.065 & 0.015 & 0.003 & 0.933 \\
\hline Physical crises (0-1) & 0.111 & 0.000 & 0.104 & 0.000 & 0.120 & 0.000 \\
\hline Mental crises (0-1) & 0.300 & 0.000 & 0.323 & 0.000 & 0.269 & 0.000 \\
\hline $\begin{array}{l}\text { Decreased interest in } \\
\operatorname{sex}(0-1)\end{array}$ & 0.045 & 0.021 & 0.061 & 0.015 & 0.015 & 0.618 \\
\hline $\begin{array}{l}\text { Evaluation of sexual } \\
\text { life (order) }\end{array}$ & -0.166 & 0.000 & -0.163 & 0.000 & -0.172 & 0.000 \\
\hline R-squared & & & & & & \\
\hline
\end{tabular}

* Dichotomous variables (1-yes; 0-no); beta - standardized parameter.

\section{DISCUSSION}

The analyses concerned 2,535 Poles aged 18-65 who took part in an Internet survey conducted about 10-11 weeks after the WHO had announced the COVID-19 pandemic. The aim of the project 'Health, bonds in relationships and sexual life of Poles in the period of COVID-19' was a subjective assessment of health from four important aspects: physical, mental, social and sexual health. The current study concerns body mass, physical, mental and sexual health, all analyzed in the context of the changes that might be caused by the COVID-19 pandemic. The analyses were stratified according to the place of residence of the respondents; thus, the hypothesis was confirmed that the introduced restrictions affected the health of residents of urban and rural areas to various extents. However, the initial hypothesis of urban disadvantage was not confirmed. The place of residence did not diversify the stress level, but other factors shaped the variability of the stress index in urban and in rural areas. It was noted that the increase of body mass of female urban residents was smaller than that of female residents of rural areas. Taking other changes related to the pandemic into consideration, the findings were not favourable for urban areas, including higher level of BMI, episodes of mental crises and worse assessment of sexual life.

Undoubtedly, the pandemic has caused a significant mental burden as the source of stress [25]. Several studies demonstrated that stress shows a complex correlation with the amount of food consumed. In their survey, Torres et al. observed that $40 \%$ of the respondents consumes more calories in stressful situations, $40 \%$ of them obtained less energy from food, and in $20 \%$, the nutrition behaviour do not change [26].

The current study shows that the occurrence of a mental and psychical crisis was related to more frequent declarations of the body mass change $(\mathrm{p}<0.001)$. Previous Polish surveys proved that an increase and decrease of body mass was reported by $29.9 \%$ and $18.6 \%$ of the respondents, respectively [14]. In the current study, in $33.7 \%$ of respondents the body mass increased within 2-3 months of the pandemic in Poland, $15.6 \%$ of the respondents declared decrease of their body mass, and in $50.2 \%$ of the respondents, the body mass remained unchanged The data are similar to the findings of surveys by the National Institute for Public Health PZH National Research Institute which showed increase of body mass in $28.3 \%$, and decrease of body mass in $12.9 \%$ of the population [17]. Furthermore, the urban respondents who reported a decrease in their body mass, had the highest average stress index, compared with the change of body mass $(p<0.001)$, whereas the respondents of rural areas whose body mass increased felt the strongest stress $(\mathrm{p}<0.001)$. More women than men reported increase (38\% vs. $29.5 \%)$ and decrease (17.2\% vs. $14.1 \%$ ) of their body mass in the beginning of the COVID-19 pandemic $(\mathrm{p}<0.001)$. A younger age was also correlated with more frequent changes of body mass $(\mathrm{p}<0.001)$. Thus, particular attention should be paid to women and people under the age of 30 . Simon GE et al. showed that people with obesity present a $25 \%$ higher risk of developing disorders of mood and fear [27].

In the period of the COVID-19 pandemic, people with obesity show an increased risk of hospitalization, serious diseases and fatality [28]; therefore, people with the BMI $>30$, particularly when their body mass increased, should be provided with dietary care. In the current study, people with overweight and obesity more often declared an increase of their body mass, and more seldom did report any decrease of the weight than it was reported by people with the proper BMI ( $\mathrm{p}<0.001)$.

Obesity negatively affects sexual life, and is related to several various sexual difficulties, both in women and in men. In the case of both genders, these are difficulties with absence of orgasm, lower frequency of intercourse, lower sexual drive and lack of satisfaction [23]. In order to prevent gaining weight during the COVID-19 pandemic, strategies consisting in self-control and regular control of weight are suggested [29].

The respondents who experienced a physical or mental crisis more often declared a change in their body mass than those who did not experience such crises during the pandemic $(p<0.001)$. The pandemic shows not only adverse effects on physical health, but it also shows adverse effects on mental health, which may in turn cause several psychological effects $[3,4,5]$. Experience of mental crisis during the initial 2-3 months of the pandemic was reported by $35 \%$ of the respondents, whereas a subjective physical crisis was experienced by $50.2 \%$ of the respondents.

In their survey, Zhang et al. applied a nine-item Patient Health Questionnaire (PHQ9), and compared two mental health indices among urban and rural dwellers. In the case of anxiety, the mean index was equal to 9.15 and 8.69 , respectively. In the case of depression, the mean index decreased from 11.25 in urban to 10.57 in rural areas [30]. In the current study, urban residents also showed a higher average stress level than residents of rural areas (6.44 vs. 6.29). The experienced mental crisis was related with a higher average stress index, both in rural and urban areas $(\mathrm{p}<0.001)$. Similar results were obtained in the current study in the case of subjective feeling of physical crisis $(\mathrm{p}<0.001)$. Among the 
general population, the highest average Cohen stress index was found in single persons $(\mathrm{SD}=6.92 ; \mathrm{p}<0.001)$. Among urban residents, the highest stress index was related to the age range of $18-29$ years $(\mathrm{SD}=7.12 ; \mathrm{p}<0.001)$, whereas in rural areas they were single persons $(\mathrm{SD}=6.97 ; \mathrm{p}<0.001)$. Single people might feel loneliness more often in the period of the COVID-19 pandemic. This crisis disturbed the hitherto functioning due to quarantine and restriction in mobility or the obligation to stay at homes, which significantly affected the mental health of the society [31].

The current study confirms the relation of stress level with demographic features and the general health condition. People with lower education showed a higher average stress level than people with secondary and university education $(\mathrm{p}<0.001)$, both in urban $(\mathrm{p}=0.135)$ and in rural areas $(p<0.001)$. Women showed a higher average stress level during the COVID-19 pandemic than men $(\mathrm{p}=0.001)$. This may have been due to factors such as combining office work from home with household responsibilities, including raising children and helping them with remote school work. Attention was also paid to people with chronic conditions. The outbreak of the pandemic affected critically also mental health of the patients who may feel symptoms of awe, fear and lack of hope regarding their treatment results [32]. The current study shows that occurrence of a chronic disease was related to a higher average stress index $(\mathrm{p}<0.001)$.

The pandemic also affected sexual life [33]. Li W et al. in their observational study showed that during the pandemic the number of sexual partners decreased [34], and an Australian cross-sectional research proved decreased sexual activity in the period of the pandemic [35]. The current study shows that the decrease of interest in sexual activity during the pandemic was experienced by $16.5 \%$ of respondents. Those who reported decreased interest in sex gained weight more often $(\mathrm{p}=0.006)$, which is compatible with surveys that show that overweight and obesity negatively affect sexual health [23].

The sexual life of Poles has been regularly surveyed since 1997 [36], and this study, shows that 18\% of Polish men and women assess their sexual life as bad. People who declared decreased interest in sexual activity showed a higher average stress index than persons who did not notice such a decrease (SD 7.48 vs. $6.15 ; \mathrm{p}<0.001$ ), whereas a higher average stress index was related to a negative assessment of the respondents' sexual life $(\mathrm{p}<0.001)$, both in urban and rural areas $(\mathrm{p}<0.001)$. During the pandemic, many women suddenly experienced a situation when, apart from on-line work, they had to take care of their children and do the housework at the same time. Such a situation may cause stress, fatigue, and - at the same time - it may result in worsening of the quality of sexual life $[37,38]$.

The presented study was cross-sectional, and it was therefore difficult to draw conclusions concerning cause and effect relationships; however, the form of asking a question about a change alleviates this drawback. From the point of view of causes of a body mass change, the lack of analyses concerning physical activity and nutritional behaviour was a limitation in the study. Only body mass change was observed as a result of living in the pandemic. The direction of the correlation was also determined conventionally as a reversible influence of stress on body mass and on sexual life, which are the key variables identified in multi-factor analyses.

\section{CONCLUSIONS}

The initial months of the COVID-19 pandemic affected the residents of urban and rural areas to different extents. Body mass changes and indicators of sexual health are important predictors of the stress level, even following the correction of the analyses for other covariates. Urban residents more often declared an increase of body mass than rural residents. An increase or decrease of body mass was mostly reported by people with $\mathrm{BMI} \geq 30$, and respondents who experienced a mental or physical crisis. Urban residents felt higher stress levels than rural residents, as indicated by the average Cohen stress index. Variability of the stress level was influenced by such factors as age, education, changes of body mass, physical or mental crisis, decrease in sexual activity, and a negative assessment of sexual life. A change of body mass did not show any significant correlation with the assessment of sexual life, whereas its correlation with the level of stress appeared significant.

\section{REFERENCES}

1. Regulation of the Minister of Health of 20 March 2020 on the declaration of an epidemic in the territory of the Republic of Poland. (DzU z 2020 r. poz. 491). Polish. (access: 2021.08.14)

2. Moccia L, Janiri D, Pepe M, et al. Affective temperament, attachment style, and the psychological impact of the COVID-19 outbreak: an early report on the Italian general population. Brain Behav Immun. 2020; 87: 75-79. doi: 10.1016/j.bbi.2020.04.048

3. Pfefferbaum B, North CS. Mental Health and the Covid-19 Pandemic. N Engl J Med. 2020; 383(6): 510-512. doi: 10.1056/NEJMp2008017

4. Brooks SK, Webster RK, Smith LE, et al. The psychological impact of quarantine and how to reduce it: rapid review of the evidence. Lancet. 2020; 395(10227): 912-920. doi: 10.1016/S0140-6736(20)30460-8

5. Pierce M, Hope H, Ford T, et al. Mental health before and during the COVID-19 pandemic: a longitudinal probability sample survey of the UK population. Lancet Psychiatry. 2020; 7(10): 883-892. doi: 10.1016/ S2215-0366(20)30308-4

6. Kumar A, Nayar KR. COVID 19 and its mental health consequences. J Ment Health. 2021; 30(1): 1-2. doi: 10.1080/09638237.2020.1757052

7. Yau YH, Potenza MN. Stress and eating behaviors. Minerva Endocrinol. 2013; 38(3): 255-67.

8. Maniscalco JW, Kreisler AD, Rinaman L. Satiation and stress-induced hypophagia: examining the role of hindbrain neurons expressing prolactin-releasing Peptide or glucagon-like Peptide 1. Front Neurosci. 2012; 6: 199. doi: 10.3389/fnins.2012.00199

9. Razzoli M, Bartolomucci A. The Dichotomous Effect of Chronic Stress on Obesity. Trends Endocrinol Metab. 2016; 27(7): 504-515. doi: 10.1016/j.tem.2016.04.007

10. Maniscalco JW, Rinaman L. Interoceptive modulation of neuroendocrine, emotional, and hypophagic responses to stress. Physiol Behav. 2017; 176: 195-206. doi: 10.1016/j.physbeh.2017.01.027

11. Spinosa J, Christiansen P, Dickson JM, Lorenzetti V, Hardman CA. From Socioeconomic Disadvantage to Obesity: The Mediating Role of Psychological Distress and Emotional Eating. Obesity (Silver Spring). 2019; 27(4): 559-564. doi: 10.1002/oby.22402

12. Almandoz JP, Xie L, Schellinger JN, et al. Impact of COVID-19 stay-athome orders on weight-related behaviours among patients with obesity. Clin Obes. 2020; 10(5): e12386. doi: 10.1111/cob.12386

13. Batlle-Bayer L, Aldaco R, Bala A, et al. Environmental and nutritional impacts of dietary changes in Spain during the COVID-19 lockdown. Sci Total Environ. 2020; 748: 141410. doi: 10.1016/j.scitotenv.2020.141410

14. Sidor A, Rzymski P. Dietary Choices and Habits during COVID-19 Lockdown: Experience from Poland. Nutrients. 2020; 12(6). doi: $10.3390 /$ nu12061657

15. Ammar A, Brach M, Trabelsi K, et al. Effects of COVID-19 Home Confinement on Eating Behaviour and Physical Activity: Results of the ECLB-COVID19 International Online Survey. Nutrients. 2020; 12(6). doi: 10.3390/nu12061583

16. Phillipou A, Meyer D, Neill E, et al. Eating and exercise behaviors in eating disorders and the general population during the COVID-19 
pandemic in Australia: Initial results from the COLLATE project. Int J Eat Disord. 2020; 53(7): 1158-1165. doi: 10.1002/eat.23317

17. Wojtynia B, Goryński P, editor. Health status of Polish population and its determinants 2020 .

18. Flentje A, Obedin-Maliver J, Lubensky ME, Dastur Z, Neilands T, Lunn MR. Depression and Anxiety Changes Among Sexual and Gender Minority People Coinciding with Onset of COVID-19 Pandemic. J Gen Intern Med. 2020; 35(9): 2788-2790. doi: 10.1007/s11606-020-05970-4

19. Jacob L, Smith L, Butler L, et al. Challenges in the Practice of Sexual Medicine in the Time of COVID-19 in the United Kingdom. J Sex Med. 2020; 17(7): 1229-1236. doi: 10.1016/j.jsxm.2020.05.001

20. World Health Organization and others. Sexual health and its linkages to reproductive health: an operational approach, 2017. https://www.who. int/reproductivehealth/publications/ sexual_health/sh-linkages-rh/ en/ (access: 2021.08.14)

21. Li G, Tang D, Song B, et al. Impact of the COVID-19 Pandemic on Partner Relationships and Sexual and Reproductive Health: CrossSectional, Online Survey Study. J Med Internet Res. 2020; 22(8): e20961. doi: $10.2196 / 20961$

22. Fuchs A, Matonóg A, Pilarska J, et al. The Impact of COVID-19 on Female Sexual Health. Int J Environ Res Public Health. 2020; 17(19) doi: 10.3390/ijerph17197152

23. Larsen SH, Wagner G, Heitmann BL. Sexual function and obesity. Int J Obes (Lond). 2007; 31(8): 1189-98. doi: 10.1038/sj.ijo.0803604

24. Lee EH. Review of the psychometric evidence of the perceived stress scale. Asian Nurs Res (Korean Soc Nurs Sci). 2012; 6(4): 121-7. doi: 10.1016/j.anr.2012.08.004

25. Salari N, Hosseinian-Far A, Jalali R, et al. Prevalence of stress, anxiety, depression among the general population during the COVID-19 pandemic: a systematic review and meta-analysis. Global Health. 2020; 16(1): 57. doi: 10.1186/s12992-020-00589-w

26. Torres SJ, Nowson CA. Relationship between stress, eating behavior, and obesity. Nutrition. 2007; 23(11-12): 887-94. doi: 10.1016/j. nut.2007.08.008

27. Simon GE, Von Korff M, Saunders K, et al. Association between obesity and psychiatric disorders in the US adult population. Arch Gen Psychiatry. 2006; 63(7): 824-30. doi: 10.1001/archpsyc.63.7.824
28. Richardson S, Hirsch JS, Narasimhan M, et al. Presenting Characteristics, Comorbidities, and Outcomes Among 5700 Patients Hospitalized With COVID-19 in the New York City Area. JAMA. 2020; 323(20): 2052-2059. doi: 10.1001/jama.2020.6775

29. Laitner MH, Minski SA, Perri MG. The role of self-monitoring in the maintenance of weight loss success. Eat Behav. 2016; 21: 193-7. doi: 10.1016/j.eatbeh.2016.03.005

30. Zhang J, Zhu L, Li S, et al. Rural-urban disparities in knowledge, behaviors, and mental health during COVID-19 pandemic: A community-based cross-sectional survey. Medicine (Baltimore). 2021; 100(13): e25207. doi: 10.1097/MD.0000000000025207

31. Nicola M, Alsafi Z, Sohrabi C, et al. The socio-economic implications of the coronavirus pandemic (COVID-19): A review. Int J Surg. 2020; 78: 185-193. doi: 10.1016/j.ijsu.2020.04.018

32. Hossain MM, Sultana A, Purohit N. Mental health outcomes of quarantine and isolation for infection prevention: a systematic umbrella review of the global evidence. Epidemiol Health. 2020; 42: e2020038. doi: 10.4178/epih.e2020038

33. Arafat SMY, Alradie-Mohamed A, Kar SK, Sharma P, Kabir R. Does COVID-19 pandemic affect sexual behaviour? A cross-sectional, crossnational online survey. Psychiatry Res. 2020; 289: 113050. doi: 10.1016/j. psychres.2020.113050

34. Li W, Li G, Xin C, Wang Y, Yang S. Challenges in the Practice of Sexual Medicine in the Time of COVID-19 in China. J Sex Med. 2020; 17(7): 1225-1228. doi: 10.1016/j.jsxm.2020.04.380

35. Coombe J, Kong FYS, Bittleston H, et al. Love during lockdown: findings from an online survey examining the impact of COVID-19 on the sexual health of people living in Australia. Sex Transm Infect. 2021; 97(5): 357-362. doi: 10.1136/sextrans-2020-054688

36. Izdebski Z. Health and sexual life of Poles in 2017. Research study in the background since 1997. Wydawnictwo Uniwersytetu Warszawskiego Warsaw, 2020; 1 .

37. Hamilton LD, Meston CM. Chronic stress and sexual function in women. J Sex Med. 2013; 10(10): 2443-54. doi:10.1111/jsm.12249

38. Wang C, Pan R, Wan X, et al. Immediate Psychological Responses and Associated Factors during the Initial Stage of the 2019 Coronavirus Disease (COVID-19) Epidemic among the General Population in China. Int J Environ Res Public Health. 2020; 17(5). doi: 10.3390/ijerph17051729 